\title{
Blocking CCR7 at the Ocular Surface Impairs the Pathogenic Contribution of Dendritic Cells in Allergic Conjunctivitis
}

\author{
Simona Schlereth, ${ }^{* \dagger}$ Hyun Soo Lee, ${ }^{* \dagger}$ \\ Payal Khandelwal, ${ }^{* \dagger}$ and Daniel R. Saban ${ }^{\star \dagger}$ \\ From the Schepens Eye Research Institute,* Boston; and the \\ Department of Ophthalmology, ${ }^{\dagger}$ Harvard Medical School, Boston, \\ Massachusetts
}

CCR7 plays a key role in mobilizing tissue dendritic cells (DCs) to the lymphoid compartment for consequent elicitation of adaptive immunity. Interfering with CCR7 function therapeutically would therefore be anticipated to inhibit the progression of atopic conditions, for example, allergic conjunctivitis (AC). However, the CCR7-CCL19/CCL21 system in the ocular surface is poorly understood as is the precise role of DCs in AC immunopathogenesis. $T$ cells from ovalbumin (OVA)-primed mice were adoptively transferred into wild-type (WT) hosts. Exogenous WT $\left(\mathrm{eGFP}^{+}\right)$versus $\mathrm{CCR}^{-/-}$DCs were engrafted subconjunctivally (SCJ), and hosts were challenged with OVA (Texas-Red+) eye drops. AC immunopathogenesis was evaluated via clinical examinations, infiltration of mast cells and eosinophils, Th2 reactivity, and serum IgE levels. AC was also assessed in actively immunized mice challenged with OVA eye drops containing $1 \%$ anti-CCR7 antibody or isotype control. In eye-draining lymph nodes (LNs), $\mathrm{OVA}^{+} \mathrm{SCJ}$ engrafted WT DCs conferred upregulated CCR7 and caused augmentation of clinical signs. This result was corroborated by increased conjunctival infiltration, Th2 cytokines in LNs, and serum OVA-specific IgE. Strikingly, this was completely reversed with SCJ engrafted $\mathrm{CCR}^{-1-}$ DCs in all parameters tested. Furthermore, topical antibody blockade of CCR7 in actively immunized mice significantly inhibited AC. Ocular surface DCs via CCR7 expression contribute to the immunopathogenesis of AC, thereby allowing significant inhibition of this experimental condition via topical CCR7 antibody blockade. (Am J Patbol 2012, 180:2351-2360; http://dx.doi.org/10.1016/j.ajpath.2012.02.015)
By capture and subsequent presentation of antigen $(\mathrm{Ag})$ to $\mathrm{T}$ helper (Th) lymphocytes, as well as being involved in driving $T$ cell differentiation (eg, Th1, Th2, and Th17), dendritic cells (DCs) play a central role in the elicitation of adaptive immune responses. Unparalleled expression of $\mathrm{MHC}$ and costimulatory molecules (eg, B7.1 and B7.2) allows mature DCs to be potent $\mathrm{T}$ cell stimulators. Likewise, DCs play a key role in activating T cells involved in allergic immunity, ${ }^{1-8}$ for example in atopic dermatitis, ${ }^{1}$ allergic rhinitis, ${ }^{2,3}$ and perhaps best characterized in allergic asthma. ${ }^{4-8}$ It has been shown that DCs are involved both at the level of Th2 sensitization to allergen, ${ }^{4-7}$ as well as in the progression of an allergic reaction and secondary immune responses via restimulation of effector/memory T cells. ${ }^{8}$ Indeed, degranulation and release of, for example, histamine and prostaglandins, makes activated mast cells and eosinophils important immune effectors in allergy. However, release of these proinflammatory factors by such leukocytes largely depends on direct and/or indirect help afforded by Th2 cells. ${ }^{9,10}$

Although sensitization and differentiation of Th cells (eg, Th2 in allergy) requires Ag presentation by DCs, engagement of these two cell types for this to occur is not a simple matter. This is in part because mature DCs that have captured Ag from inflamed tissue sites must mobilize to the lymphoid compartment where large pools of $T$ cells are found. One way in which this mobilization process is facilitated involves the CCR7-CCL19/21 chemokine axis. ${ }^{11,12}$ Mature DCs upregulate CCR7 at the inflamed tissue site and thereby respond chemotactically to a CCL21 gradient established by local lymphatic endothelial cells-a process that facilitates entry into lymphatics and consequently into the regional lymph node $(\mathrm{LN}) .{ }^{13,14}$ Furthermore, via CCL21 expression by high endothelial venules (HEV), which are positioned within

Supported in part by the Lions Club of Massachusetts (D.R.S.) and the Eleanor and Miles Shore Fellowship of Harvard Medical School (D.R.S.).

Accepted for publication February 9, 2012

Supplemental material for this article can be found at http://ajp. amjapthol.org or at http://dx.doi.org/10.1016/j.ajpath.2012.02.015.

Address reprint requests to Daniel R. Saban, Ph.D., 20 Staniford St., Boston, MA 02117. E-mail: daniel.saban@schepens.harvard.edu. 
the T cell-rich paracortex, CCR7 also helps traffic DCs toward T cells within the $\mathrm{LN} .{ }^{11-14}$

However, whether CCR7 expression on DCs at the ocular surface would promote allergic Th2 responses in allergic conjunctivitis $(A C)$, requires further investigation. First, a role for DCs in AC has yet to be directly determined, ${ }^{15}$ although previous reports have demonstrated $\mathrm{DC}$ infiltration in $\mathrm{AC} .^{2}$ Second, other systems of chemotaxis including VEGFR3, have been implicated in mobilization from the ocular surface to LNs in the initiation of adaptive immune responses. ${ }^{16-18}$ In contrast, CCR7 in ocular surface DCs has demonstrated to have a tolerogenic role, as shown in the corneal transplant model. ${ }^{19}$ Likewise, numerous other reports have shown in allergic asthma models that the CCR7-CCL19/21 axis is associated with the mediation of tolerance induction to allergen exposure. ${ }^{20-23}$ For example, Hintzen et $\mathrm{al}^{23}$ reported a defect in DCs of CCR7 ${ }^{-1-}$ mice, but not in wild-type mice, in a model of airway-induced tolerance. In addition, Yamashita et $\mathrm{al}^{21}$ and Grinnan et $\mathrm{al}^{22}$ independently reported exaggerated allergen-induced airway inflammation in plt mutant mice (ie, deficient in lymphoid CCL21 and CCL19).

We therefore set out to examine the specific role in $\mathrm{AC}$ of CCR7 expression by DCs at the level of the ocular surface, and to determine what effect blockade of CCR7 expression would have on the immunopathogenesis of this condition. We addressed this important question in an established model of AC. ${ }^{24}$ Similar to the approach previously reported in an allergic asthma model ${ }^{5-7,25}$ we evaluated allergy immunopathogenesis to exogenous DCs engrafted within the conjunctiva. This allowed the examination of the CCR7-CCL19/CCL21 system specifically on DCs in the ocular surface to allergen challenges. Data described herein demonstrate that whereas exogenous DCs augmented the progression of AC immunopathogenesis, these increased responses were completely abrogated in mice administered CCR7 knockout DCs. Furthermore, this effect was mirrored when CCR7 antagonizing antibody $(\mathrm{Ab})$ was administered topically. We therefore uncovered a novel strategy, involving CCR7 blockade, which can potentially be applied clinically for management of $\mathrm{AC}$, and our results indicate this to be based on an important role for CCR7 expression by ocular surface DCs in promoting allergic immune responses.

\section{Materials and Methods}

\section{Mice and Anesthesia}

C57BL/6 male mice 8 to 12 weeks old were purchased from Charles River Laboratories (Wilmington, MA). CCR7 knockout mice (on a C57BL/6 background) were provided by Andrew Luster (Massachusetts General Hospital, Boston, MA) and Martin Lipp (Maz-Delbrueck-Center of Molecular Medicine, Berlin, Germany). Mice were housed in a specific pathogen-free environment at the Schepens Eye Research Institute animal facility. All procedures were approved by the Institutional Animal
Care and Use Committee, and all animals were treated according to the ARVO Statement for the Use of Animals in Ophthalmic and Vision Research. Anesthesia was used for all surgical procedures with i.p. administered ketamine/xylazine suspensions (120 and $20 \mathrm{mg} /$ $\mathrm{kg}$, respectively).

\section{Generation of Bone Marrow-Derived DC}

Bone marrow-derived DC (BMDC) were generated as previously described. ${ }^{26}$ Briefly, femurs and tibiae were collected from freshly euthanized mice. BM cells were seeded at $2 \times 10^{5} / \mathrm{mL}$ in RPMI 1640 (BioWitthaker, Walkersville, MD) supplemented with 10\% FBS, 1\% penicillin/ streptomycin, plus $20 \mathrm{ng} / \mathrm{mL}$ of mouse GM-CSF (Biolegend, San Diego, $\mathrm{CA}$ ) at $37^{\circ} \mathrm{C}$ with $5 \% \mathrm{CO}_{2}$. Medium was changed on day 4 , and nonadherent and loosely adherent cells were collected on day 7 and thoroughly washed.

\section{T-Cell Adoptive Transfer}

$\mathrm{T}$ cells, for adoptive transfer, were obtained from donor wild-type (WT) C57BL/6 mice that were immunized once i.p. with a $100-\mu \mathrm{L}$ suspension containing $1 \mathrm{mg}$ of aluminum hydroxide (Sigma-Aldrich, St. Louis, MO) diluted in HBSS, $300 \mathrm{ng}$ of pertussis toxin (Sigma-Aldrich), and 100 $\mu \mathrm{g}$ of ovalbumin (Sigma-Aldrich). Donor mice 2 weeks post immunization, a time-point consistent with high OVAspecific IgE titers in the sera (data not shown), were euthanized and spleens were collected. Donor spleens were prepared into single-cell suspensions by tissue press using a sterile $70-\mu \mathrm{m}$ sieve, and cells were then treated with red blood cell lysis buffer according to manufacturer's instructions (Sigma-Aldrich) and washed thoroughly. Donor $\mathrm{T}$ cells were enriched for via magnetic activated cell sorting using anti-CD90.2 Ab according to manufacturer's instructions (Miltenyi Biotec, Auburn, MA). The sorted donor population was then enumerated via trypan blue exclusion assay, and donor T cells were set at a concentration $1 \times 10^{7} / \mathrm{mL}$ of sterile HBSS. Recipient mice were adoptively transferred IV with $1 \times 10^{6}$ donor T cells.

\section{AC Induction}

OVA-primed T cells were prepared as described above, and $1 \times 10^{6} \mathrm{~T}$ cells were adoptively transferred into naive hosts. Host mice were then anesthetized 16 hours later for unilateral injection of cells into the subconjunctival space as previously described. ${ }^{27}$ Injection volume was $10 \mu \mathrm{L}$ of sterile HBSS and contained $1 \times 10^{5}$ BMDCs. Challenge via topical OVA instillation $(250 \mu \mathrm{g} / 5$ $\mu \mathrm{L}$ eye drop) was administered immediately following subconjunctival (SCJ) injection, and then challenged additionally (to account for significant tearing post SCJ injection) twice more in 20-minute intervals. Challenges were subsequently administered once daily for at least 10 days. In some experiments, fluorescein isothiocyanate (FITC)- or Texas Red-conjugated OVA (Sigma-Aldrich) was used for topical challenges. 


\section{AC Clinical Scoring}

This procedure has been described previously ${ }^{28}$ and was performed here in a masked fashion by two independent observers. Briefly, scoring was performed 20 minutes post challenge and done once daily from day 1 (ie, 24 hours after SCJ injection) to at least day 10. Mice where examined biomicroscopically based on four independent parameters, which include lid swelling, tearing, chemosis, and conjunctival vasodilation (redness). Each parameter was ascribed 0 (ie, absent) to $3+$ points (ie, maximal) and was summed to yield a maximum score of $12+$.

\section{OVA-Specific IgE Quantitation}

Blood was collected and kept at room temperature to allow coagulation. Serum was separated via centrifugation and then collected and pooled. Aliquoted samples were immediately stored at $-30^{\circ} \mathrm{C}$. Aliquots were thawed at room temperature and diluted serially from 1:5 to 1:25. Samples were analyzed in duplicate or triplicate using a 96-well format using an OVA-specific mouse IgE ELISA (enzyme-linked immunoabsorbent assay) Kit (AbD Serotec, Raleigh, NC) performed according to the manufacturer's instructions.

\section{Conjunctival Mast Cell and Eosinophil Quantitation}

After 20 minutes following topical challenge, mice were euthanized, and surgically procured conjunctivae (including bulbar through palpebral regions from both the superior and inferior areas) were placed on ice cold PBS. Single-cell suspensions were prepared using standard collagenase digestion methods as previously described. ${ }^{29}$ Briefly, conjunctivae were minced into small fragments, followed by digestion with $2 \mathrm{mg} / \mathrm{mL}$ of collagenase type IV and $0.5 \mathrm{mg} / \mathrm{mL}$ of DNase I (Roche, Basel, Switzerland) for 2 to 3 hours at $37^{\circ} \mathrm{C}$. The suspension was triturated using a syringe and filtered through a $70-\mu \mathrm{m}$ cell strainer. Cells were thoroughly washed and resuspended in $0.5 \%$ BSA buffer, followed by an anti-FcR (CD16/CD32) blockade step as per the manufacturer's instructions (BD Pharmingen, San Diego, CA). Cells were subsequently stained as per the manufacturer's instructions with Alexa-647-conjugated anti-CD45 Ab (Biolegend), Alexa-488-conjugated anti-CD117 (Biolegend), PE-conjugated CCR3 (Biolegend), or appropriate isotype controls. After 30 minutes, cells were washed and resuspended for subsequent acquisition using a BD LSR II flow cytometer (BD Biosciences, San Jose, CA).

\section{Measurement of T Cell Responses to Recall Allergen Stimulation}

Regional LNs (cervical and submandibular) were collected and pooled from freshly euthanized mice. Singlecell suspensions were prepared and T cells were MACS sorted as described above. Enriched T cells were enumerated via trypan blue exclusion assay, and plated in round-bottomed, 96-well dishes at a concentration of $1.25 \times 10^{6} / \mathrm{mL}$. Immature BMDCs prepared as described above were plated with $\mathrm{T}$ cells at a concentration $0.625 \times$ $10^{6} / \mathrm{mL}$. Co-cultures were plated in triplicate wells of RPMI (10\% FBS) with OVA (1 mg/mL) for up to 24 hours and restimulated with PMA/lonomycin and Brefeldin A (SigmaAldrich) for up to 9 hours. Harvested supernatant was measured via ELISA for Th2 (IL-4, IL-5, and IL-13) cytokines, as per the manufacturer's instructions (Ready-Set-Go ELISA Kit; eBioscience, San Diego, CA).

\section{RNA Isolation and Real-Time PCR}

Total RNA was isolated using Trizol (Invitrogen, Grand Island, NY) and RNeasy Microkit (Qiagen, Valencia, CA). The first strand of complementary DNA (cDNA) was synthesized with random hexamers using SuperScript IIITM Reverse Transcriptase (Invitrogen), and quantitative realtime PCR was performed using Taqman Universal PCR Mastermix and FAM-MGB dye-labeled predesigned primers (Applied Biosystems, Carlsbad, CA) for CCR7 (Mm01301785_m1), CCL19 (Mm00839967_g1), CCL21 (Mm03646971_gH), and glyceraldehydes 3-phosphate dehydrogenase (GAPDH) (Mm99999915_g1). A 2- $\mu$ L quantity of cDNA was loaded in each well, and the assays were performed in duplicate. The GAPDH gene was used as the endogenous reference for each reaction. The results were analyzed by the comparative threshold cycle (CT) method, and the relative expression level of each sample was expressed as fold change from naïve control.

\section{Topical CCR7 Blockade}

WT C57BL/6 mice were immunized once i.p. with a $100-\mu \mathrm{L}$ suspension containing $1 \mathrm{mg}$ aluminum hydroxide (Sigma-Aldrich) diluted in HBSS, $300 \mathrm{ng}$ pertussis toxin (Sigma-Aldrich), and $100 \mu \mathrm{g}$ ovalbumin (Sigma-Aldrich). Three weeks post immunization, mice were challenged topically, once daily for 4 consecutive days, with OVA (250 $\mu \mathrm{g})$-loaded eye drops (5 $\mu \mathrm{L})$ containing 1\% monoclonal $\mathrm{Ab}(25 \mu \mathrm{g})$ against CCR7 or the matching isotype control (R\&D Systems).

\section{Statistical Analysis}

Statistical analyses included one-way analysis of variance and Bonferroni's multiple comparison test, in addition to two-tailed Student's $t$-test. SE and SD of the mean were calculated. A $P$ value $<0.05$ was considered statistically significant.

\section{Results}

\section{DCs from Allergen-Exposed Ocular Surface Confer Up-Regulated CCR7 Expression in Lymphoid Tissues}

We began by investigating whether CCR7 expression by DCs from allergen-exposed tissue-sites (ie, conjunctiva) plays a role in the mobilization of these cells to the lym- 
phoid compartment in an allergic immune response. To accomplish this, we locally administered exogenous eGFP $^{+}$DCs SCJ via injection into WT mice, and immediately thereafter instilled eye drops of Texas-Red-conjugated OVA, thus allowing us to identify migrating allergen-laden DCs (ie, Texas-Red+ eGFP ${ }^{+}$) in host LN. Exogenous DCs used here were derived from bone marrow cells of syngeneic eGFP ${ }^{+}$hosts and cultivated via standard ex vivo procedures. ${ }^{26}$ Bone marrow-derived DCs were well suited for our line of investigation, as use of these cells in a similar manner has been previously been described, ${ }^{25}$ and also we found these DCs to be highly efficient at capturing OVA in vitro, as well as effective at stimulating Th2 expansion in vitro (see Supplemental Figure S1, A and B, at http://ajp.amjpathol.org).

Mice used in this experiment were adoptively transferred with OVA/Alum-primed T cells and subsequently challenged via a series of instillations with OVA-loaded eye drops to induce AC (Figure 1A). Mice were subsequently rested for several weeks, and then received SCJ injection with eGFP ${ }^{+}$DCs plus immediate stimulation with conjugated OVA eye drops (Figure 1A), thus mimicking initiation of an allergic reaction. Regional LNs (ie, cervical and submandibular LN) including the active ipsilateral node and the presumably inactive contralateral node were then harvested for fluorescence-activated cell sorter (FACS) analyses. This was also performed in nonAC-induced control mice as well, which are indicated in Figure 1B. Data in Figure 1 represent analyses at 4 hours post challenge, as conjugated OVA was less detectable at later time-points, including 12 and 24 hours. mRNA analysis was also conducted on conjunctival tissues of immunized subsequent to challenge, and a fold increase of $1.870 \pm$ 0.2660 in CCR7 $(P=0.04)$ and $1.714 \pm 0.1527$ in CCL19 $(P=0.001)$, but not in CCL21 $(P=0.8)$, relative to naive conjunctiva was observed (data not shown).

In the LNs, we detected a very clear OVA+ CD11c+ population unique to the ipsilateral LNs of $A C$-induced mice (Figure 1B). This was in contrast to a marginal population of $\mathrm{OVA}^{+} \mathrm{CD}_{11 \mathrm{C}^{+}} \mathrm{DCs}$ observed in the contralateral LNs of these mice (Figure 1B), and similarly observed in LNs of both groups of non-AC-induced controls (Figure 1B). We also tested AC-induced recipients of topical conjugated-OVA stimulation but without SCJ-injected DCs; however, the frequency of $\mathrm{OVA}^{+} \mathrm{DCs}$ was eightfold lower than in similar mice with SCJ-injected DCs and topical OVA stimulation (see Supplemental Figure $\mathrm{S} 1, \mathrm{C}$ and $\mathrm{D}$, at $h$ ttp://ajp.amjpathol.org). This verified the mobilization of SCJ-injected DCs to local ipsilateral LNs in our model. Furthermore, in AC-induced mice, recipients that were SCJ injected with eGFP+ DCs and stimulated with topical OVA, we found that nearly $80 \%$ were eGFP $^{+}$within the OVA ${ }^{+} \mathrm{CD}_{11 \mathrm{c}^{+}}$population (Figure 1B). Strikingly, this was twofold higher than the frequency of eGFP+ DCs found in the ipsilateral LNs of non-AC-induced mice that received equivalent OVA topical challenges; and eightfold higher than in non-AC-induced mice that did not receive OVA topical challenges (Figure 1B). Finally, nearly all eGFP ${ }^{+}$DCs found in ipsilateral LNs of $\mathrm{AC}$-induced mice were $\mathrm{CCR} 7^{+}$(Figure $1 \mathrm{C}$ ), and these levels were clearly increased relative to their original ex-
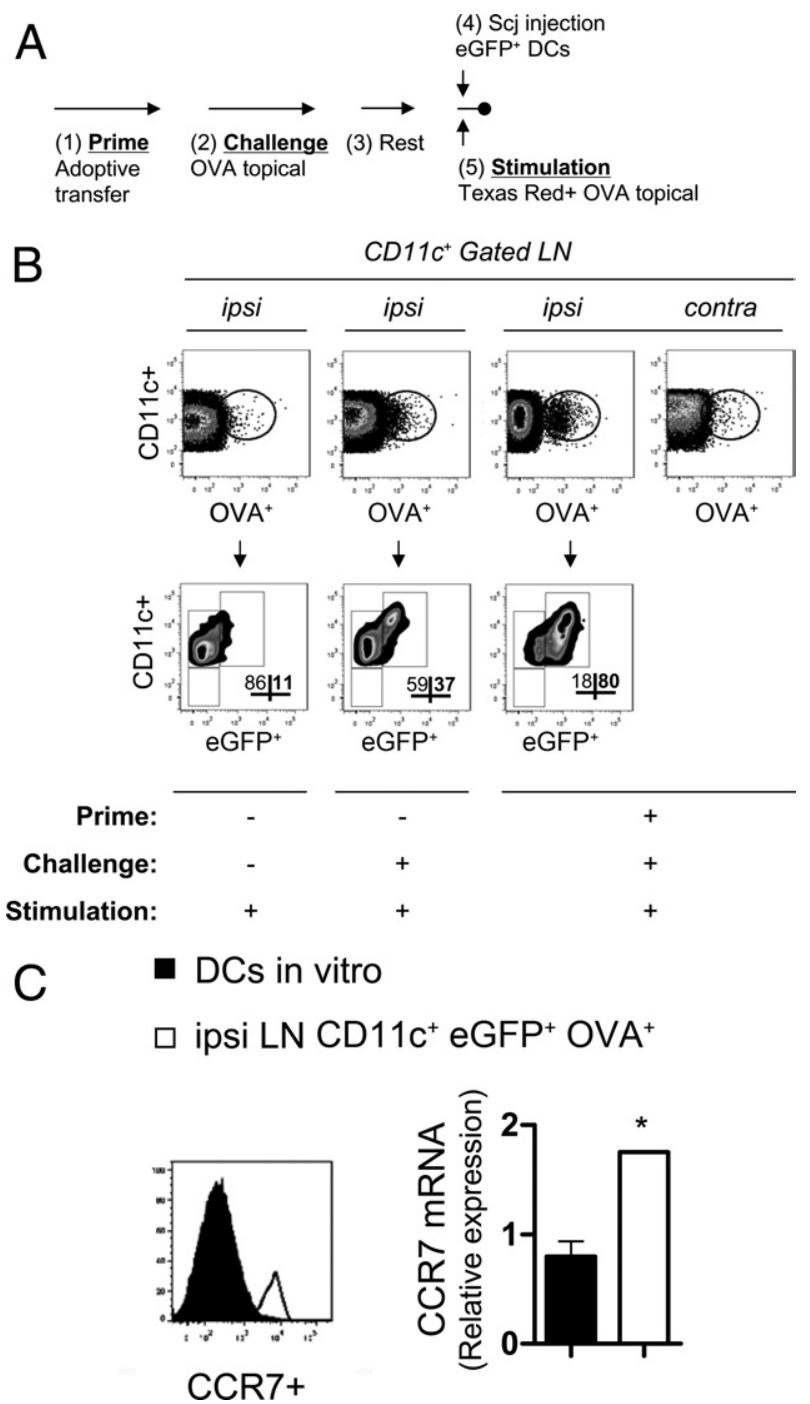

Figure 1. DCs mobilized from ocular surface confer increased CCR7 expression in the lymphoid compartment of AC-induced mice. A: MACS-sorted T cells harvested from OVA/Alum-primed mice were adoptively transferred into naive hosts (1). Subsequently hosts were challenged once daily for at least 10 days via topical instillation of OVA-loaded drops to one eye (2). Mice were then rested for several weeks (3), and subsequently SCJ injected with $\mathrm{eGFP}^{+}$DCs (4), followed by immediate stimulation with Texas Red-conjugated OVA eye drops (5). B: Identification of SCJ-injected eGFP+ DCs that are $\mathrm{OVA}^{+}$in ipsilateral LNs of AC-induced hosts. FACS analysis of DCs was performed on ipsilateral (ipsi) and contralateral (contra) LNs. OVA ${ }^{+} \mathrm{CD}_{11 \mathrm{c}^{+}}$ cells were gated on and subsequently $\mathrm{eGFP}^{+} \mathrm{CD} 11 \mathrm{c}^{+}$frequencies were enumerated. C: SCJ-injected $\mathrm{eGFP}^{+} \mathrm{DCs}$ that are $\mathrm{OVA}^{+}$confer increased CCR7 expression in LNs of AC-induced mice. By flow cytometry and realtime PCR, in vitro generated $\mathrm{eGFP}^{+}$DCs prior SCJ injection were directly compared with CD11c ${ }^{+} \mathrm{eGFP}^{+} \mathrm{OVA}^{+}$DCs found in ipsi LNs of AC-induced mice. Each experimental group represents $n=3$ hosts; data are representative of multiple independent experiments.

pression levels seen in vitro before SCJ injection (Figure 1C). Taken together, these data suggest that in AC-induced mice, allergen-laden DCs mobilized to regional LNs confer increased CCR7 expression.

\section{DCs from Ocular Surface Mount Th2 Responses in CCR7-Dependent Fashion}

Our findings suggested that upregulated CCR7 by allergen-laden DCs is associated with optimal mobilization of 

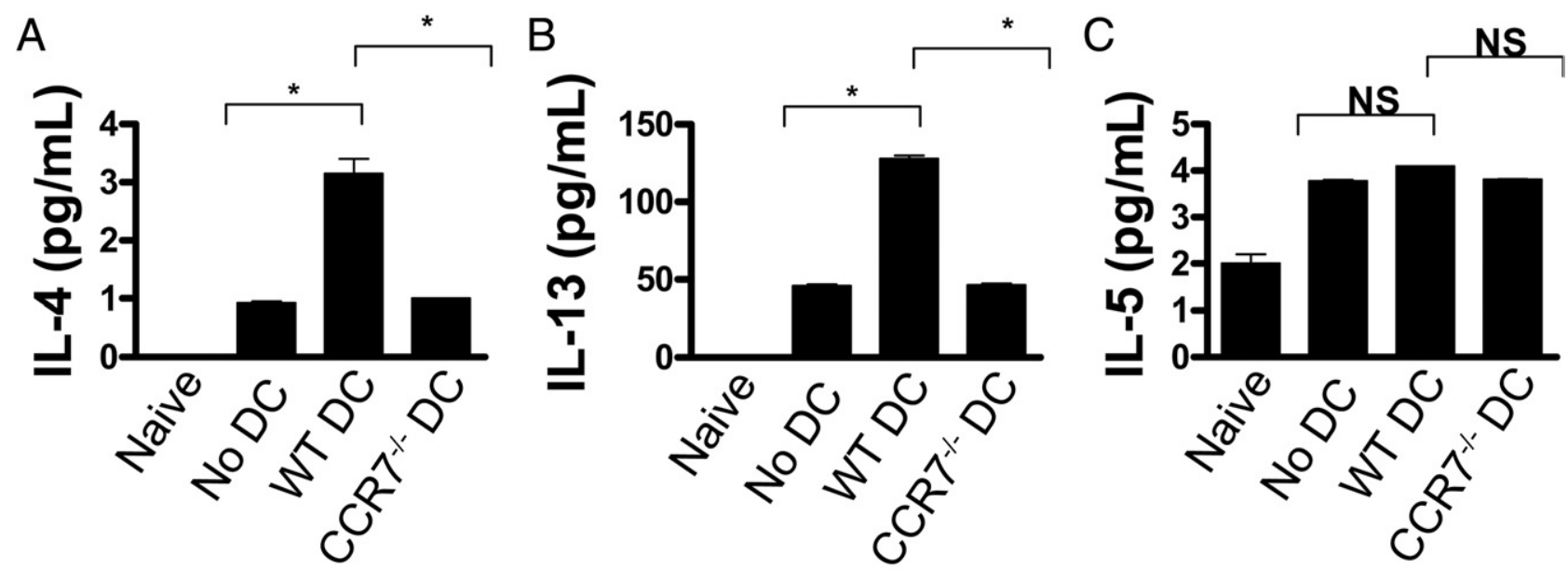

Figure 2. DCs from the ocular surface mount allergen-reactive Th2 responses in a CCR7-dependent manner. WT DCs, CCR $7^{-/-}$DCs, or no SCJ injection was administered into mice after adoptive transfer with OVA/Alum-primed T cells. After at least 10 days of once daily OVA topical challenges, host T cells were harvested from ipsi LNs and recall stimulated in vitro with OVA. Control wells consisted of T cells from naive mice stimulated with OVA in vitro as well. Supernatant was analyzed via ELISA for IL-4 (A), IL-13 (B), and IL-5 (C). Data are derived from $n=5$ hosts per group; figure represents multiple independent experiments. $\left({ }^{*} P<0.05\right)$

these cells to lymphoid tissues in an allergic response. However, once in the LN, whether these DCs are relevant in stimulating Th2 remains unknown. To address this, we established in vitro recall (ie, OVA) stimulation assays of $T$ cells isolated from ipsilateral LNs of AC-induced mice. Furthermore, to allow us to assess the role on Th2 responses of CCR7 expression by DCs from allergen-exposed sites, T cells for these experiments were harvested from AC-induced mice influenced by SCJ injection of WT versus $\mathrm{CCR} 7^{-1-}$ DCs. A similar approach with injected mast cells in $\mathrm{AC}$ was reported by Fukeda et $\mathrm{al},{ }^{27}$ as well as injection of exogenous DCs previously described by Lambrecht et al ${ }^{5,6}$ and others in the allergic asthma model. ${ }^{7,25}$

Mice were adoptively transferred with OVA/Alumprimed T cells, shortly thereafter were SCJ injected with WT versus $\mathrm{CCR}^{-1-} \mathrm{DCs}$, and then immediately challenged with instillations of OVA-loaded eye drops to induce AC. MACS-sorted T cells from ipsilateral LNs were then restimulated in vitro to OVA, and ELISA was used to measure Th2 cytokine levels (eg, IL-4, IL-5, and IL-13). Using this system, we found augmented levels of $\mathrm{IL}-4$ and IL-13 in T cells isolated from AC-induced mice SCJ injected with WT DCs (Figure 2, A and B). Strikingly, augmentation was completely reversed in $\mathrm{AC}$-induced mice that received $\mathrm{CCR} 7^{-1-} \mathrm{DCs}$, as IL-4 and IL-13 returned to control levels; ie, AC-induced mice that did not receive SCJ injected with WT DCs. No significant differences in IL-5 levels were observed among the experimental groups (Figure 2C).

OVA-specific IgE in the sera of AC-induced mice SCJ injected with WT versus $\mathrm{CCR} 7^{-1-}$ DCs was also assessed, as Th2 cytokines (including IL-13 and IL-4) are required for consequent plasma cell synthesis of IgE. We found that control AC-induced mice, which were not SCJ injected with DCs, had marginal to low levels of OVA specific IgE, whereas these levels were augmented nearly 10fold in mice that were SCJ injected with WT DCs (Figure 3). However, this augmentation was completely reversed, to levels seen in controls, when AC-induced mice were SCJ injected instead with CCR7 ${ }^{-1-}$ DCs (Figure 3), suggesting that DCs from allergen-exposed sites mount allergen Th2 responses in in a CCR7-dependent fashion.

\section{DCs from Ocular Surface Contribute via CCR7 to Elicitation of Allergic Conjunctivitis}

Th2 cytokines are important in eosinophil degranulation and allergen-specific IgE is important in mast cell degranulation, which cause an allergic reaction. Based on this understanding, the suggested CCR7-dependent mechanism by which DCs mounts a Th2 response, and consequent synthesis of IgE, would therefore be expected to lead to an allergic reaction as well. Thus, to determine whether this might be the case, adoptively

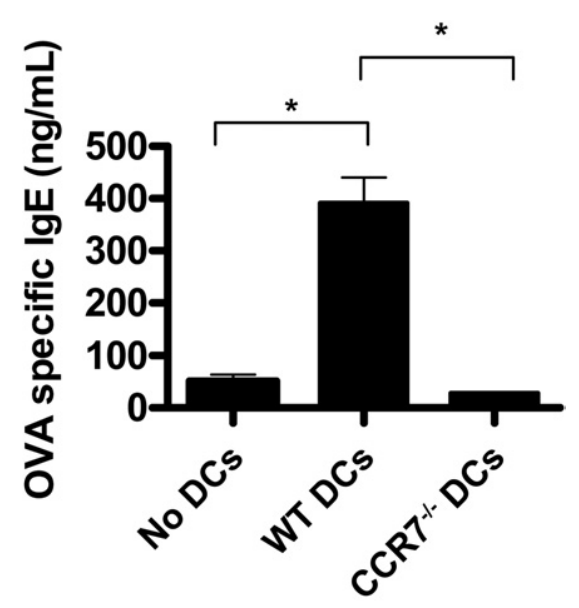

Figure 3. Expression of CCR7 by DCs from the ocular surface is necessary for contribution to consequent IgE production. WT DCs, CCR $7^{-/-}$DCs, or no SCJ injection was administered to mice following adoptive transfer with OVA/Alum-primed T cells. Blood was collected from hosts after at least 10 days of once daily OVA topical challenges. Sera were isolated and measured for OVA-specific IgE. Data are derived from $n=5$ hosts per group; figure represents multiple independent experiments. ( $\left.{ }^{*} P<0.05\right)$ 
transferred mice received an SCJ injection of WT or $\mathrm{CCR} 7^{-1-} \mathrm{DCs}$, and the onset and progression of $\mathrm{AC}$ clinical signs to topical OVA challenges were examined. Challenges were administered once daily for more than 10 days and scored 20 minutes post challenge as previously described. ${ }^{28}$ In doing so, we observed that SCJ injection of WT DCs to adoptively transferred mice significantly augmented AC clinical scores as early as day 5 of topical challenge $(P<0.05)$, relative to adoptively transferred mice that received no SCJ injection or sham PBS SCJ injection (Figure 4, A and B). However, this augmentation was completely abrogated when OVA challenges were instilled on adoptively transferred hosts that were SCJ injected with CCR7 ${ }^{-1-}$ DCs (Figure 4, A and B), suggesting that DCs from allergen-exposed sites contribute to an allergic reaction in a CCR7-dependent manner.

We also enumerated mast cells and eosinophils in the conjunctivae of AC-induced mice influenced by SCJ injection of WT versus CCR7 ${ }^{-1-}$ DCs, as allergic reactivity can correlate with increased infiltration of mast cells and eosinophils. FACS analysis was therefore performed on collagenase-digested conjunctivae of AC-induced mice topically challenged for at least 10 days (a time point consistent with peak AC clinical signs). Mast cells were discerned by their $\mathrm{CD}_{4} 5^{+} \mathrm{C}-\mathrm{Kit}^{+}$phenotype, as confirmed by the P815 mastocytoma cell line, which were $\sim 100 \% \mathrm{CD}^{2} 5^{+} \mathrm{c}-\mathrm{Kit}^{+}$(data not shown). We found that baseline frequencies of mast cells were $0.3 \%$ or less in the normal conjunctivae (data not shown), whereas peak AC clinical signs increased to $1.4 \% \mathrm{CD}^{2} 5^{+} \mathrm{C}-\mathrm{Kit}^{+}$(Figure 4C). In AC-induced mice SCJ injected with WT DCs, this was increased to $2.2 \% \mathrm{CD}^{4} 5^{+} \mathrm{C}-\mathrm{Kit}^{+}$(Figure $4 \mathrm{C}$ ), thus corroborating the augmented scores seen clinically in these mice. However, this increase was completely abrogated to $1.2 \% \mathrm{CD}_{4} 5^{+} \mathrm{C}-\mathrm{Kit}^{+}$in $\mathrm{AC}$-induced mice SCJ injected with $\mathrm{CCR} 7^{-1-}$ DCs (Figure $4 \mathrm{C}$ ). This trend was consistent when enumerating eosinophils (CD45+ CCR3+) as well, as AC-induced mice recipient of SCJinjected WT DCs had increased eosinophils, whereas this increase was completely reversed in mice recipient of SCJ-injected CCR7 ${ }^{-1-}$ DCs (Figure 4C).

\section{Subconjunctival Injection with WT, but Not $C C R 7^{-\prime-}$, DCs Augments Allergic Immune Responses in CCR7 ${ }^{-1-}$ Hosts}

We next determined whether CCR7-sufficient endogenous DCs could significantly influence the effect seen by SCJ-injected WT or CCR7 ${ }^{-1-}$ DCs in our AC model. We accomplished this by using CCR $7^{-1-}$ hosts (thereby consisting of CCR7 ${ }^{-1-}$ endogenous DCs) and compared the effect of SCJ-injected WT versus CCR7 ${ }^{-1-}$ DCs on allergic immunity in our AC model. Mice were adoptively transferred with OVA/Alum-primed T cells (WT), followed by SCJ injection of DCs and immediate topical challenge. Mice were challenged once daily for more than 10 days and clinically scored. Conjunctivae were also harvested for FACS enumeration of mast cells and eosinophils, and blood was collected for OVA-specific IgE quantitation of sera via ELISA.

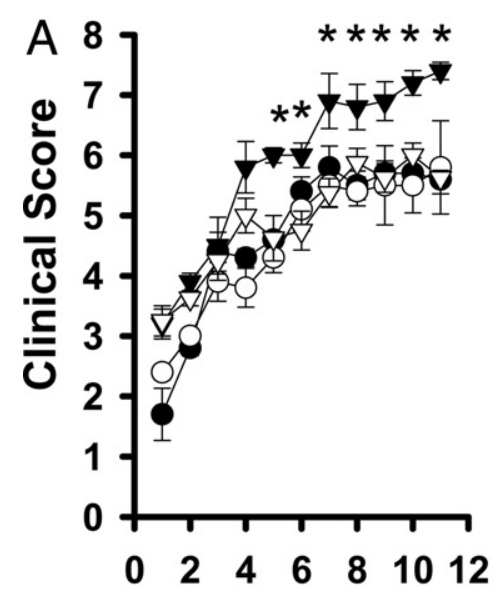

- No SCJ

O Sham SCJ

$\nabla$ WT DC SCJ $\nabla \mathrm{CCR}^{-/ \mathrm{DC}} \mathrm{SCJ}$

\section{Challenge Days}

B

Naïve WT DCs $\quad$ CCR7 7 DCs

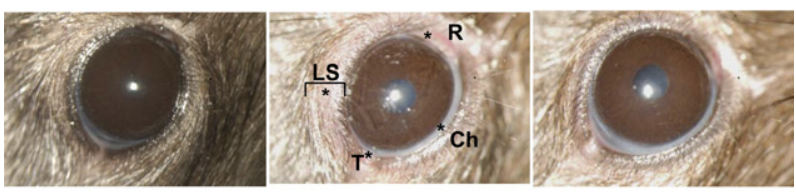

C Conjunctivae
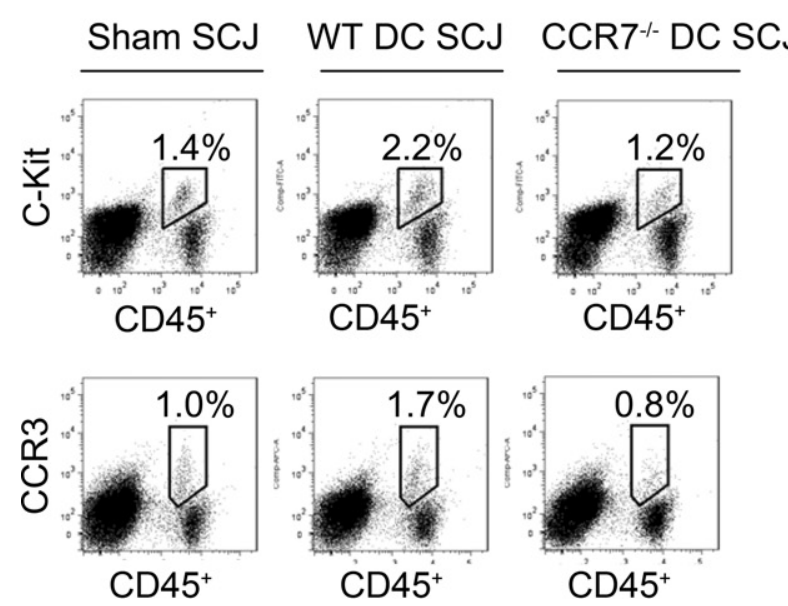

Figure 4. DCs from the ocular surface lead to AC in a CCR7-dependent fashion. A: SCJ injection of $\mathrm{CCR} 7^{-1-}$ DCs results in impaired onset and progression of AC clinical signs. WT DCs, CCR $7^{-/-}$DCs, sham HBSS, or no SCJ injection was administered into mice after adoptive transfer with OVA Alum-primed T cells. All mice were challenged topically with OVA eye drops once daily for at least 10 days and were scored biomicroscopically 20 minutes post challenge for clinical signs [eg, redness (R), chemosis (Ch), tearing/ discharge (T), and lid swelling (LS)]. B: Representative clinical photographs of mice SCJ administered with WT versus CCR $7^{-1-}$ DCs on day 10 of topical challenge. C: Impaired AC clinical scores conferred by CCR $7^{-1-}$ DCs are associated with decreased number of conjunctival mast cells and eosinophils. Conjunctivae were collected from AC-induced mice after at least 10 days of once daily OVA topical challenges. Tissue was prepared into single-cell suspensions, and FACS analysis was performed for mast cells (CD $\left.45^{+}{\mathrm{C}-\mathrm{Kit}^{+}}^{+}\right)$ and eosinophils $\left(\mathrm{CD} 45^{+} \mathrm{CCR}^{+}\right)$. Data are derived from $n=4$ to 5 hosts per group; figure represents multiple independent experiments.

In $\mathrm{CCR} 7^{-1-}$ hosts, SCJ injection of WT DCs augmented clinical scores as early as day 1 relative to control $\mathrm{CCR} 7^{-1-}$ mice that were not SCJ injected with DCs (Figure 5A). This was not the case in AC-induced $\mathrm{CCR} 7^{-1-}$ 
mice that were SCJ injected with $\mathrm{CCR} 7^{-1-} \mathrm{DCs}$, as clinical scores were only slightly and mostly insignificantly elevated over those of control mice (Figure 5A). In an additional experiment, we adoptively transferred OVA/

A

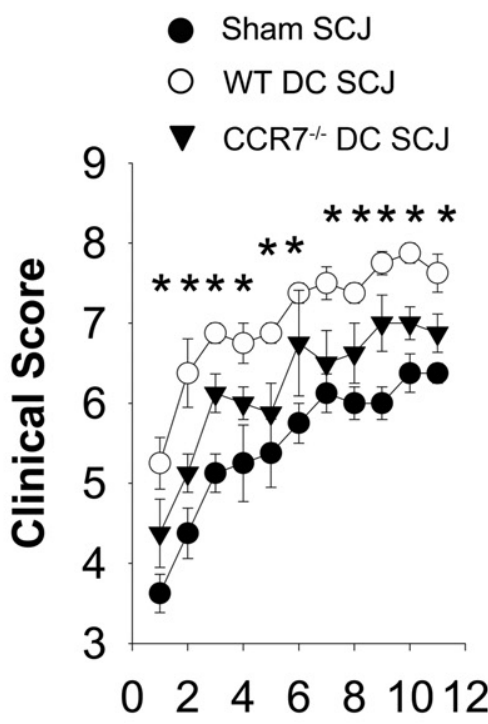

Challenge Days

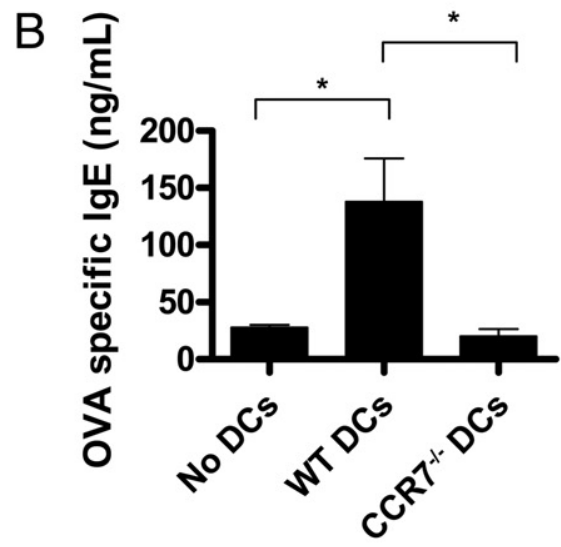

C

\section{Conjunctivae}
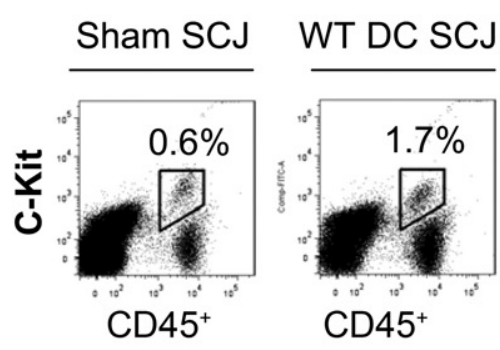

CCR7-^DC SCJ
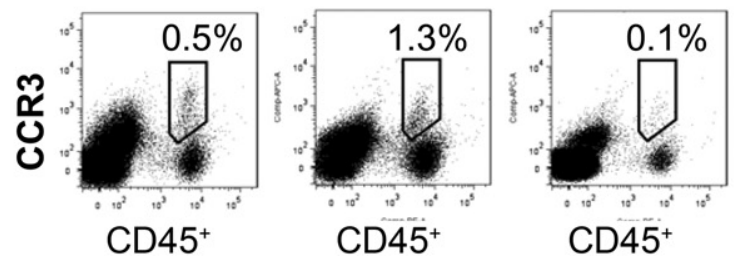

Alum-primed OTII T cells into CCR7 $7^{-1-}$ hosts (as performed by Hintzen et $\mathrm{al}^{23}$ ), and again found that SCJ injection of WT DCs, but not CCR7 ${ }^{-1-}$ DCs, augmented clinical signs in AC (see Supplemental Figure S1E at http://ajp.amjpathol.org). Quantitation of OVA-specific IgE similarly showed augmented levels in SCJ-injected mice with WT DCs, relative to control mice that were not SCJ injected (Figure 5B), whereas in SCJ-injected mice with $\mathrm{CCR} 7^{-1-}$ DCs, levels were not augmented and were similar to that of the control (Figure 5B). In line with this, mast cell $\left(\mathrm{CD} 45^{+} \mathrm{C}^{-\mathrm{Kit}^{+}}\right)$and eosinophil $\left(\mathrm{CD} 45^{+} \mathrm{CCR} 3^{+}\right)$ numbers were augmented only in SCJ-injected mice with WT DCs, relative to control (Figure $5 \mathrm{C}$ ); whereas this augmentation was absent in SCJ-injected mice with $\mathrm{CCR}^{-1-}$ DCs (Figure 5C). Thus, SCJ-injected wild-type, but not $\mathrm{CCR}^{-1-}$, DCs augments allergic immune responses in $\mathrm{CCR} 7^{-1-}$ hosts.

\section{Examination of Topical Administration of Anti- CCR7 Ab Blockade in Allergic Conjunctivitis}

Because CCR7-deficient DCs at the level of the ocular surface demonstrated an impaired capacity to trigger allergic immune responses to OVA instilled eye drops, we sought to determine whether we can achieve a similar effect clinically with topically administered anti-CCR7 Ab in actively immunized mice. To test this, mice were immunized against OVA and then rested for 3 weeks. Mice were subsequently challenged once daily for 4 days with OVA eye drops containing either $1 \%$ anti-CCR7 Ab or isotype control Ab (Figure 6). We measured clinical scores at 20 minutes, 6 hours and 24 hours post challenge (Figure 6). We found that at all three of these time points post challenge, anti-CCR7 Ab administration significantly decreased clinical scores, particularly to challenges given on days $3(P<0.05)$ and $4(P<0.05)$ (Figure 6A).

\section{Discussion}

The current study uses a novel strategy, involving topical administration of CCR7 antagonist, which our results indicate can potentially be applied clinically for management of allergic conjunctivitis. We found the efficacy of this blockade to be based on an important role for CCR7 expression by ocular surface DCs in promoting allergic

Figure 5. SCJ injection of WT, but not $\mathrm{CCR} 7^{-/-}$, DCs augments allergic immune responses in CCR $7^{-1-}$ hosts. A: SCJ-injected WT DCs augment AC clinical signs in $\mathrm{CCR} 7^{-1-}$ hosts. WT DCs, CCR $7^{-/-}$DCs, or sham HBSS were SCJ injected into mice after adoptive transfer with OVA/Alum-primed T cells (WT). All mice were challenged topically with OVA eye drops once daily and scored for at least 10 days. B: SCJ-injected WT DCs contribute to increased OVA-specific IgE to in CCR $7^{-/-}$hosts. Blood was collected from hosts after at least 10 days of once daily OVA topical challenges. Sera were isolated and measured for OVA-specific IgE. C: SCJ-injected WT DCs lead to augmented conjunctival mast cells and eosinophils in $\mathrm{CCR}^{-/-}$hosts. Conjunctivae were collected from AC-induced mice after at least 10 days of once daily OVA topical challenges and prepared for FACS analysis of mast cells (CD $45^{+}$

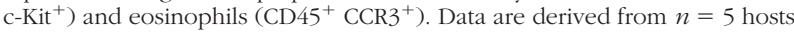
per group; figure represents multiple independent experiments. ( $\left.{ }^{*} P<0.05\right)$ 


\section{A $\square$ Isotype control Ab}

\section{А Anti-CCR7 Ab}

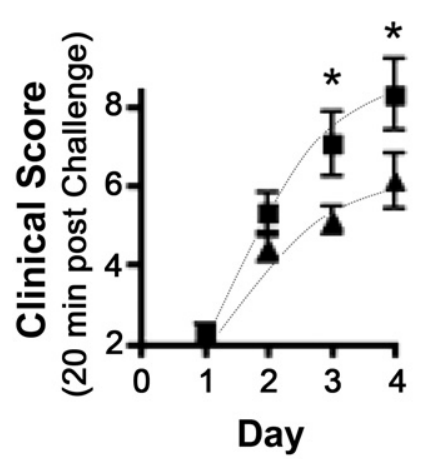

B
Day 3

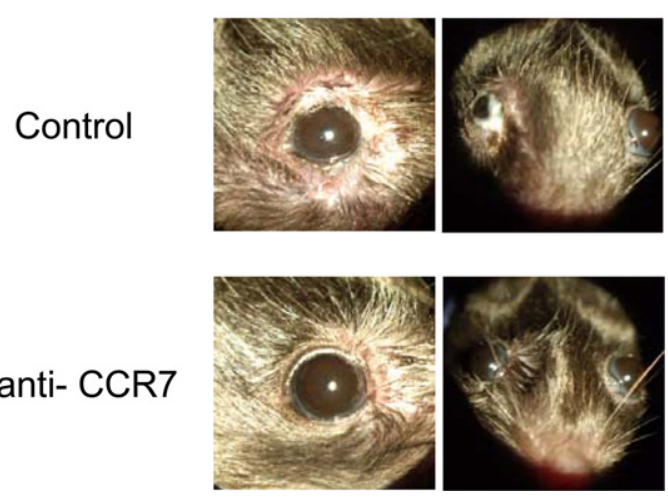

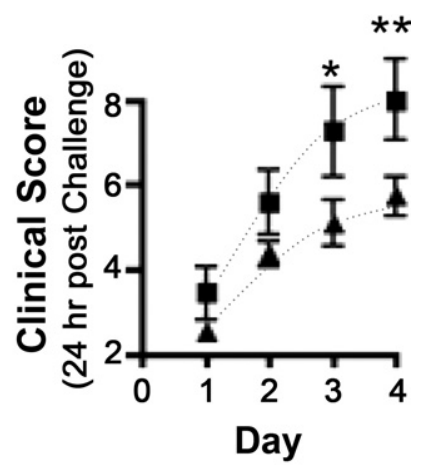

Day 4
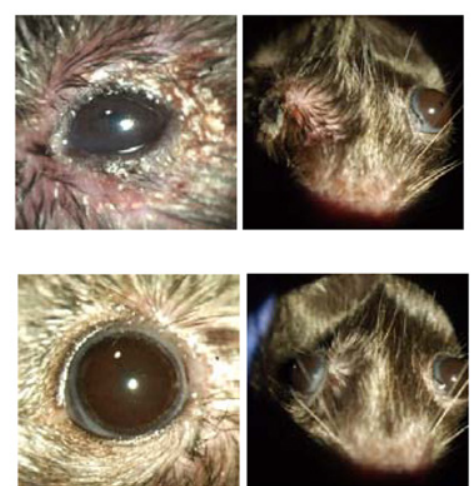

Figure 6. AC clinical signs are decreased by administration of topical CCR7 Ab blockade. Actively immunized mice were challenged once daily for 4 days with OVA eye drops containing $1 \%$ anti-CCR7 Ab or isotype control Ab. A: Mice were scored for clinical signs biomicroscopically at 20 minutes, 6 hours, and 24 hours post challenge. B: Representative clinical images on challenge days 3 and 4 are presented. Data are derived from $n=5$ hosts per group; figure represents multiple independent experiments. $\left({ }^{*} P<0.05\right)$

immune responses. These findings to some extent are in contrast to previous work describing a role for DC expression of CCR7 in the cornea ${ }^{19}$ (and also shown in the lung ${ }^{20-23}$ ) in the suppression of immunity via tolerance. These potentially opposing roles (ie, promoting versus modulating immunity) highlight the notion that CCR7 function on DCs has various facets, and thereby underscores the need for studies such as these to continue to elucidate its important function in controlling immune responses. ${ }^{20}$

The role of CCR7 in promoting AC immunopathogenesis was revealed in an experimental murine model in which WT mice were engrafted with exogenous WT versus $\mathrm{CCR}^{-1-}$ DCs into the conjunctiva. This approach is in contrast to inducing allergy in $\mathrm{CCR} 7^{-1-}$ mice or plt mice $^{21-23}$ (ie, mice deficient in lymphoid CCL19 and CCL21) to address this, as lymphocytes (in addition to DCs) also express CCR7. Our approach enabled a focus on the function of the CCR7-CCL19/CCL21 axis on DCs from the ocular surface. Furthermore, exogenous DCs were not allergen pulsed before engraftment, and akin to endogenous tissue DCs, thus had to capture allergen from topical exposure. This is distinguishable from seminal work done in allergic airway hypersensitivity, whereby exogenous DCs were pulsed with allergen before intratracheal injection. ${ }^{5-7,25}$ Hence, the model herein allowed us to determine the effect of deleting CCR7 in DCs at the ocular surface, and in doing so we found a stark impairment in AC immunopathogenesis.

Several pieces of evidence allowed making the novel identification that the CCR7-CCL19/CCL21 axis plays a key role in mobilizing ocular surface DCs to generate an allergic immune response. This is supported by our observation that nearly all SCJ-injected DCs $\left(\mathrm{eGFP}^{+}\right)$that had captured topically instilled OVA (Texas Red+) conferred very strong upregulation of $\mathrm{CCR}^{+}$expression in the LNs of AC-induced mice. Furthermore, mobilization of SCJ-injected eGFP ${ }^{+}$DCs to LNs was optimal in mice that were both allergen primed and subsequently allergen challenged (both of which are required to induce an allergic reaction in $\mathrm{AC}$ ), thus implicating a role for an allergic reaction in triggering DC mobilization. In contrast, only marginal mobilization of SCJ-injected eGFP ${ }^{+}$DCs to LNs was found in non-AC-induced mice (ie, without an allergic reaction), including mice that were primed but not challenged, or challenged but not primed.

These series of experiments, in addition to demonstrating optimal mobilization of DCs from the site of allergen exposure to $L N$, also signified a potentially important difference by which allergen is processed in allergy. Our data suggest that in AC-induced mice, allergen is cap- 
tured and presumably presented by DCs from the ocular surface. In contrast, in non-AC-induced mice, "free"-form allergen in lymphatic drainage ${ }^{30,31}$ is likely captured (and presumably presented) mostly by other DCs (eGFP-), such as LNs resident DCs. This is supported by the observation that less than $40 \%$ of OVA+ DCs were $\mathrm{eGFP}^{+}$in LNs of non-AC-induced mice; whereas $80 \%$ of allergen-laden DCs were eGFP ${ }^{+}$in $\mathrm{LNs}$ of AC-induced mice. The significance of this may be related to the findings of Hawiger et $\mathrm{al}^{32}$ who reported that resident LNs DCs (in contrast to mobilized tissue DCs) presented Ag in a tolerogenic fashion. ${ }^{32}$ Future work, however, is required to determine whether this difference in allergen processing plays a role, at least in part, in how reactivity to allergen exposure varies between atopic and non-atopic individuals.

In addition to facilitating entry into regional lymphatics and LN, CCR7 could also play an important role in directing allergen-laden DCs to the T cell-rich paracortex region of the LNs in allergy. Indeed, this process is made possible by the CCL21 gradient established by HEV Iocated in the paracortex, ${ }^{11-14}$ and would promote engagement and activation of T cells by DCs. ${ }^{11-14}$ Consistent with this, we found that in vitro stimulated (with recall allergen) $\mathrm{T}$ cells isolated from $\mathrm{AC}$-induced mice that were SCJ injected with WT DCs showed a significantly enhanced Th2 reaction, as indicated by IL-13 and IL-4 (but not IL-5) production. This augmented Th2 reactivity was completely abrogated in $\mathrm{T}$ cells isolated from AC-induced mice SCJ injected with CCR7 ${ }^{-1-}$ DCs. Thus, our finding - namely, that DCs that capture allergen from the ocular surface lead to Th2 responses in a CCR7-dependent fashion-is likely contributed in part by CCR7-mediated trafficking of DCs to the paracortex, to promote Th2 in turn.

Consistent with this, our additional findings indicate that production of allergen-specific IgE is also led to by DCs from ocular surface in a CCR7-dependent fashion. This is supportive evidence because differentiation of Ag-experienced B cells, a process that is required for development of IgE synthesizing plasma cells, is mostly driven by Th2 cytokines. ${ }^{33}$ Thus, as seen in AC-induced mice that were SCJ injected with WT DCs, augmented Th2 responses could be expected to have caused the observed increase in allergen-specific IgE production (via promoting differentiation of allergen-experienced B cells). Furthermore, consistent with Roozendaal et $\mathrm{al}^{31}$ and others, ${ }^{32} \mathrm{~B}$ cells likely captured allergen (a prerequisite for their differentiation) in the free form within lymphatic drainage.

The totality of our findings, indicating that DCs from the ocular surface mount allergic immune responses in a CCR7-dependent fashion, are not in conflict with the understanding that the CCL19/21-CCR7 axis can also be involved with mediating allergen-induced tolerance. ${ }^{21-23}$ Indeed, CCR7 expressed by different immune cells is known to exert different effects. ${ }^{20}$ For instance, $\mathrm{CD} 4{ }^{+} \mathrm{CD} 25^{+} \mathrm{FoxP}^{+}$Treg expression of CCR7 is known to facilitate the trafficking of these cells into LNs for consequent suppression of effector T cell expansion. ${ }^{34}$ Also relevant are reports that implicate an impaired Treg com- partment in plt and $\mathrm{CCR}^{-1-}$ mice, ${ }^{13,34}$ which thereby could have contributed to the impaired tolerance induction to allergen seen in $\mathrm{CCR}^{-1-}$ mice by Hintzen et $\mathrm{al}^{23}$ or the exaggerated allergen responses observed by $\mathrm{Ya}$ mashita et $\mathrm{al}^{21}$ and Grinnan et al ${ }^{22}$ in plt mice. ${ }^{21,22}$ Likewise reflected in our study, SCJ injection of WT DCs led to significant augmentation of $A C$ clinical signs in $\mathrm{CCR} 7^{-1-}$ mice as early as day 1 of allergen challenge, but not until day 5 in WT hosts. Thus, in interpreting the sum of others' and ours findings, it is very important to consider the specific immune cell population that is being affected when examining the CCR7-CCL19/21 axis in any immune response. ${ }^{20}$

Areas that need to be further investigated involve delineating at what level(s) ocular surface CCR7 blockade inhibits allergic immunity. For example, does CCR7 blockade primarily mediate this via inhibition of ocular surface DCs from gaining access to the lymphatics, and/or downstream activities such as intranodal trafficking to the T cell rich paracortex? Another aspect that requires further investigation is how remnant antigen presentation may occur in the absence of CCR7. For example, are ocular surface DCs in the CCR7 blockade setting migrating to the LNs via other chemokine species such as CCR8-CCL1, or are other antigen presenting cells such as macrophages capable of this?

In summary, we conclude that blockade of CCR7 at the level of the ocular surface inhibits the immunopathogenesis of AC. Our data suggest that blockade interferes with the mobilization of allergen-laden DCs to regional LNs and their trafficking specifically to the paracortex for engagement of cognate $T$ cells. This in turn inhibits Th2 reactivity and consequent synthesis of IgE, both of which contribute to the allergic reaction, as indicated by this AC model. This is consistent with the report by Hammad et $\mathrm{al}^{35}$ that CCR7 expression by DCs promotes allergic pulmonary inflammation, and such convergent findings in the both ocular surface and lung thus give further credence to the importance of CCR7 expression by DCs in eliciting allergic immunity. Future development of strategies in using CCR7 antagonists may therefore hold significant therapeutic value in the management of allergic conjunctivitis and other forms of atopic disease.

\section{References}

1. Novak N, Koch S, Allam JP, Bieber T: Dendritic cells: bridging innate and adaptive immunity in atopic dermatitis [Review]. J Allergy Clin Immunol 2010, 125:50-59

2. Ohbayashi M, Manzouri B, Flynn T, Toda M, Ikeda Y, Nakamura T, Ono SJ: Dynamicchanges in conjunctival dendritic cell numbers, anatomical position and phenotype during experimental allergic conjunctivitis. Exp Mol Pathol 2007, 83:216-223

3. KleinJan A, Willart M, van Rijt LS, Braunstahl GJ, Leman K, Jung S Hoogsteden HC, Lambrecht BN: An essential role for dendritic cells in human and experimental allergic rhinitis. J Allergy Clin Immunol 2006, 118:1117-1125

4. Soumelis V, Reche PA, Kanzler H, Yuan W, Edward G, Homey B, Gilliet M, Ho S, Antonenko S, Lauerma A, Smith K, Gorman D, Zurawski S, Abrams J, Menon S, McClanahan T, de Waal-Malefyt RR, Bazan F, Kastelein RA, Liu YJ: Human epithelial cells trigger dendritic cell mediated allergic inflammation by producing TSLP. Nat Immunol 2002, 3:673-680 
5. Lambrecht BN, De Veerman M, Coyle AJ, Gutierrez-Ramos JC, Thielemans K, Pauwels RA: Myeloid dendritic cells induce Th2 responses to inhaled antigen, leading to eosinophilic airway inflammation. J Clin Invest 2000, 106:551-559

6. Lambrecht BN, Pauwels RA, De Fazekas St Groth B: Induction of rapid $T$ cell activation, division, and recirculation by intratracheal injection of dendritic cells in a TCR transgenic model. J Immunol 2000, 164:2937-2946

7. Sung S, Rose CE, Fu SM: Intratracheal priming with ovalbumin- and ovalbumin 323-339 peptide-pulsed dendritic cells induces airway hyperresponsiveness, lung eosinophilia, goblet cell hyperplasia, and inflammation. J Immunol 2001, 166:1261-1271

8. van Rijt LS, Jung S, Kleinjan A, Vos N, Willart M, Duez C, Hoogsteden $\mathrm{HC}$, Lambrecht BN: In vivo depletion of lung CD11c+ dendritic cells during allergen challenge abrogates the characteristic features of asthma. J Exp Med 2005, 201:981-991

9. Finkelman FD, Katona IM, Urban JF Jr., Holmes J, Ohara J, Tung AS, Sample JV, Paul WE: IL-4 is required to generate and sustain in vivo IgE responses. J Immunol 1988, 141:2335-2341

10. Wills-Karp M, Luyimbazi J, Xu X, Schofield B, Neben TY, Karp CL, Donaldson DD: Interleukin-13: central mediator of allergic asthma. Science 1998, 282:2258-2261

11. Sozzani S, Allavena P, D'Amico G, Luini W, Bianchi G, Kataura M, Imai T, Yoshie O, Bonecchi R, Mantovani A: Differential regulation of chemokine receptors during dendritic cell maturation: a model for their trafficking properties. J Immunol 1998, 161:1083-1086

12. Dieu MC, Vanbervliet B, Vicari A, Bridon JM, Oldham E, Aït-Yahia S, Brière $F$, Zlotnik $A$, Lebecque $S$, Caux $C$ : Selective recruitment of immature and mature dendritic cells by distinct chemokines expressed in different anatomic sites. J Exp Med 1998, 188:373-386

13. Gunn MD, Kyuwa S, Tam C, Kakiuchi T, Matsuzawa A, Williams LT, Nakano $\mathrm{H}$ : Mice lacking expression of secondary lymphoid organ chemokine have defects in lymphocyte homing and dendritic cell localization. J Exp Med 1999, 189:451-460

14. Saeki H, Moore AM, Brown MJ, Hwang ST. Cutting edge: secondary lymphoid-tissue chemokine (SLC) and CC chemokine receptor 7 (CCR7) participate in the emigration pathway of mature dendritic cells from the skin to regional lymph nodes. J Immunol 1999, 162 2472-2475

15. Ishida W, Fukuda K, Kajisako M, Takahashi A, Sumi T, van Rooijen N, Fukushima: Conjunctival macrophages act as antigen-presenting cells in the conjunctiva during the development of experimental allergic conjunctivitis. Mol Vis 2010, 16:1280-1285

16. Hamrah $P$, Chen L, Cursiefen $C$, Zhang $Q$, Joyce NC, Dana MR Expression of vascular endothelial growth factor receptor-3 (VEGFR-3) on monocytic bone marrow-derived cells in the conjunctiva. Exp Eye Res 2004, 79:553-561

17. Chen L, Hamrah P, Cursiefen C, Zhang Q, Pytowski B, Streilein JW, Dana MR: Vascular endothelial growth factor receptor-3 mediates induction of corneal alloimmunity. Nat Med 2004, 10:813-815

18. Hamrah $P$, Chen L, Zhang Q, Dana MR: Novel expression of vascula endothelial growth factor receptor (VEGFR)-3 and VEGF-C on cornea dendritic cells. Am J Pathol 2003, 163:57-68

19. Jin Y, Chauhan SK, Saban DR, Dana R: Role of CCR7 in facilitating direct allosensitization and regulatory $\mathrm{T}$-cell function in high-risk corneal transplantation. Invest Ophthalmol Vis Sci 2010, 51:816-821

20. Sánchez-Sánchez N, Riol-Blanco L, Rodríguez-Fernández JL: The multiple personalities of the chemokine receptor CCR7 in dendritic cells. J Immunol 2006, 176:5153-5159
21. Yamashita N, Tashimo H, Matsuo Y, Ishida H, Yoshiura K, Sato K, Yamashita N, Kakiuchi T, Ohta K: Role of CCL21 and CCL19 in allergic inflammation in the ovalbumin-specific murine asthmatic model. J Allergy Clin Immunol 2001, 117:1040-2006

22. Grinnan D, Sung SS, Dougherty JA, Knowles AR, Allen MB, Rose CE 3rd, Nakano H, Gunn MD, Fu SM, Rose CE Jr.: Enhanced allergeninduced airway inflammation in paucity of lymph node $\mathrm{T}$ cell (plt) mutant mice. J Allergy Clin Immunol 2006, 118:1234-1241

23. Hintzen G, Ohl L, del Rio ML, Rodriguez-Barbosa JI, Pabst O, Kocks JR, Krege J, Hardtke S, Förster R: Induction of tolerance to innocuous inhaled antigen relies on a CCR7-dependent dendritic cell-mediated antigen transport to the bronchial lymph node. J Immunol 2006, 177:7346-7354

24. Ozaki A, Ishida W, Fukata K, Fukushima A, Ueno H: Detection of antigen-specific $\mathrm{T}$ cells in experimental immune-mediated blepharoconjunctivitis in DO11.10 T cell receptor transgenic mice. Microbiol Immunol 2004, 48:39-48

25. Kuipers H, Soullié T, Hammad H, Willart M, Kool M, Hijdra D, Hoogsteden HC, Lambrecht BN. Sensitization by intratracheally injected dendritic cells is independent of antigen presentation by host antigen-presenting cells. J Leukoc Biol 2009, 85:64-70

26. Lutz MB, Kukutsch N, Ogilvie AL, Rössner S, Koch F, Romani N, Schuler G: An advanced culture method for generating large quantities of highly pure dendritic cells from mouse bone marrow. J Immunol Methods 1999, 223:77-92

27. Fukuda K, Ohbayashi M, Morohoshi K, Zhang L, Liu FT, Ono SJ: Critical role of IgE-dependent mast cell activation in a murine model of allergic conjunctivitis. J Allergy Clin Immunol 2009, 124:827-833

28. Reyes NJ, Mayhew E, Chen PW, Niederkorn JY: NKT cells are necessary for maximal expression of allergic conjunctivitis. Int Immunol 2010, 22:627-636

29. Saban DR, Chauhan SK, Zhang X, El Annan J, Jin Y, Dana R: 'Chimeric' grafts assembled from multiple allodisparate donors enjoy enhanced transplant survival. Am J Transplant 2009, 9:473-482

30. Pape KA, Catron DM, Itano AA, Jenkins MK: The humoral immune response is initiated in lymph nodes by $B$ cells that acquire soluble antigen directly in the follicles. Immunity 2007, 26:491-502

31. Roozendaal R, Mempel TR, Pitcher LA, Gonzalez SF, Verschoor A, Mebius RE, Von Andrian UH, Carroll MC: Conduits mediate transport of low-molecular-weight antigen to lymph node follicles. Immunity 2009, 30:264-276

32. Hawiger D, Inaba K, Dorsett Y, Guo M, Mahnke K, Rivera M, Ravetch $\mathrm{JV}$, Steinman RM, Nussenzweig MC: Dendritic cells induce peripheral T cell unresponsiveness under steady state conditions in vivo. J Exp Med 2001, 194:769-779

33. Emson CL, Bell SE, Jones A, Wisden W, McKenzie AN: Interleukin (IL)-4-independent induction of immunoglobulin (Ig)E, and perturbation of T cell development in transgenic mice expressing IL-13. J Exp Med 1998, 188:399-404

34. Schneider MA, Meingassner JG, Lipp M, Moore HD, Rot A: CCR7 is required for the in vivo function of $\mathrm{CD} 4+\mathrm{CD} 25+$ regulatory $T$ cells. J Exp Med 2007, 204:735-745

35. Hammad $H$, Lambrecht $B N$, Pochard $P$, Gosset $P$, Marquillies $P$, Tonnel AB, Pestel J: Monocyte-derived dendritic cells induce a house dust mite-specific Th2 allergic inflammation in the lung of humanized SCID mice: involvement of CCR7. J Immunol 2002, 169:1524-1534 